\title{
Hand-assisted laparoscopic liver resection using Habib's technique: early experience
}

\author{
Petr Vavra ${ }^{1,2}$, Peter Ihnat ${ }^{1}$, Michaela Vavrova ${ }^{3}$, Lubomir Martinek ${ }^{1}$, Jan Dostalik ${ }^{1}$, Nagy Habib ${ }^{2}$ \\ 1Department of Surgery, University Hospital Ostrava, Czech Republic \\ 2Department of Radiology, City Hospital Ostrava, Czech Republic \\ 3HPB Unit, Division of Surgery, Hammersmith Hospital, Imperial College London, United Kingdom
}

Videosurgery and Other Miniinvasive Techniques 2012; 7 (1): 8-12

DOI: 10.5114/wiitm.2011.25739

\begin{abstract}
Introduction: Hand-assisted laparoscopic liver surgery, a newly developed technique based on an innovative concept, has proved useful and safe for a variety of less invasive hepatectomies. Radiofrequency-assisted hepatic resection has been reported to be safe, associated with minimal morbidity and mortality and decreased intraoperative blood loss and transfusion requirements.

Aim: We describe how we perform hand-assisted laparoscopic radiofrequency-assisted hepatic resection using a bipolar radiofrequency device.

Results: The use of the hand port has allowed the surgeon to use his hand in direct liver manipulation, mobilization, and retraction. It was also useful for tactile tumour localization. Radiofrequency-assisted hepatic parenchymal transection was performed on 15 patients using a bipolar device (Habib 4X) with minimal blood loss (74 ml), and very decent operative and resection times (92 min, 33 min respectively).

Conclusions: This combined procedure offers a safe, effective and rapid liver resection technique. This might encourage surgeons to perform a minimally invasive approach for liver resection more frequently.
\end{abstract}

Key words: hand-assisted, laparoscopic liver resection, radiofrequency-assisted.

\section{Introduction}

Laparoscopic liver resection presented a unique technical challenge in the past decades. It has been looked upon with scepticism because of concerns regarding parenchymal transection, bleeding control, and bile leakage [1-3]. The laparoscopic approach had its limitations, which included difficulty in exposure and retraction, loss of tactile palpation, the risk of massive bleeding, air embolism, and the adequacy of oncological operations in cases of hepatic malignancy [4].

Most of these problems are successfully overcome with the improvement of laparoscopic technique and modification of instruments.
Hand-assisted [5, 6] laparoscopic liver resections have been shown to provide numerous advantages, while maintaining a minimally invasive approach. The hand has provided the perfect retracting instrument, facilitated adequate exposure of the pathology, assisted in mobilizing the liver from its attaching ligaments to the diaphragm [7], and provided a rapid and effective way to tamponade bleeding, should this occur [8].

The radiofrequency-assisted technique for liver resection (in open surgery) has been reported to be associated with minimal blood loss, a low blood transfusion requirement, and reduced mortality and morbidity rates $[9,10]$. 
Herein, we describe how we perform a handassisted laparoscopic radiofrequency-assisted liver resection.

\section{Material and methods}

Between May 2007 and December 2009, overall 49 patients with primary and secondary liver tumours underwent radiofrequency-assisted hepatic resection at the University Hospital Ostrava, Czech Republic.

During the study period, we performed handassisted laparoscopic radiofrequency-assisted hepatic resection in 15 patients ( 9 male and 6 female) with mean age of 63 years (range 51-75 years). Indications for hand-assisted laparoscopic resection, and the locations and characteristics of the lesions are summarized in Tables I and II. As shown in Table I lesions were located in right inferior segments $\mathrm{V}$ and $\mathrm{VI}$ and in left segments II and III. The types of liver resection included metastasectomy $(n=7)$, segmentectomy $(n=5)$ and bisegmentectomy $(n=3)$.

Our procedures were performed after obtaining informed consent and were performed under general endotracheal anaesthesia. The patient was placed in a modified lithotomy position. The chief surgeon stood between the patient's legs, the first assistant on the left side of the patient and the camera man on the right. After creating a pneumoperitoneum using the percutaneous technique $(12 \mathrm{mmHg})$, a $10 \mathrm{~mm}$ trocar was inserted through a sub-umbilical incision, with a $30^{\circ}$ laparoscope inserted to explore the peritoneal cavity. Two trocars were positioned: a $12 \mathrm{~mm}$ trocar in the left hypochondrium and one $5 \mathrm{~mm}$ trocar in the epigastrium (Figure 1). As many of these patients had previous abdominal surgery, adhesions were a common finding and they needed to be meticulously taken down using either laparoscopic scissors or the harmonic scalpel (Ethicon EndoSurgery, Cincinnati, Ohio). Once the liver was exposed and dissected free from adhesions, the laparoscopic ultrasonography transducer was inserted into the abdomen. Irrespective of extensive preoperative imaging, small peritoneal and intrahepatic metastases, the presence of which contradicts any major resection, still cannot be completely excluded. Therefore, routine laparoscopic staging is strongly recommended.

After exploration of the abdominal cavity and exclusion of the presence of extra-hepatic disease, an approximately 6 to $7 \mathrm{~cm}$ right transverse incision
Table I. Patients' clinical details

\begin{tabular}{|lc|}
\hline Mean age [years] & 63 (range 51-75) \\
\hline Sex (M/F) & $9 / 6$ \\
\hline Diagnosis & 10 \\
\hline Colorectal cancer metastasis & 3 \\
\hline Breast cancer metastasis & 2 \\
\hline Chronic inflammation & 38 (range: 15-53) \\
\hline Mean tumour size [mm] & 3 \\
\hline \begin{tabular}{l} 
Location of tumour \\
\hline Segment II
\end{tabular} & 5 \\
\hline Segment III & 1 \\
\hline Segment V & 5 \\
\hline Segment VI & 1 \\
\hline Segment V and VI & \\
\hline
\end{tabular}

Table II. Type and results of resection

\begin{tabular}{|lc|}
\hline Type of resection $(n)$ & 15 \\
\hline Metastasectomy & 7 \\
\hline Segmentectomy & 5 \\
\hline Bisegmentectomy & 3 \\
\hline Mean blood loss [ml] & 74 (range: 25-115) \\
\hline Mean operative time [min] & 92 (range: 45-130) \\
\hline Mean resection time [min] & 33 (range: 21-75) \\
\hline $\begin{array}{l}\text { Mean length of hospital stay } \\
\text { [days] }\end{array}$ & 5.1 (range: 4-7) \\
\hline
\end{tabular}

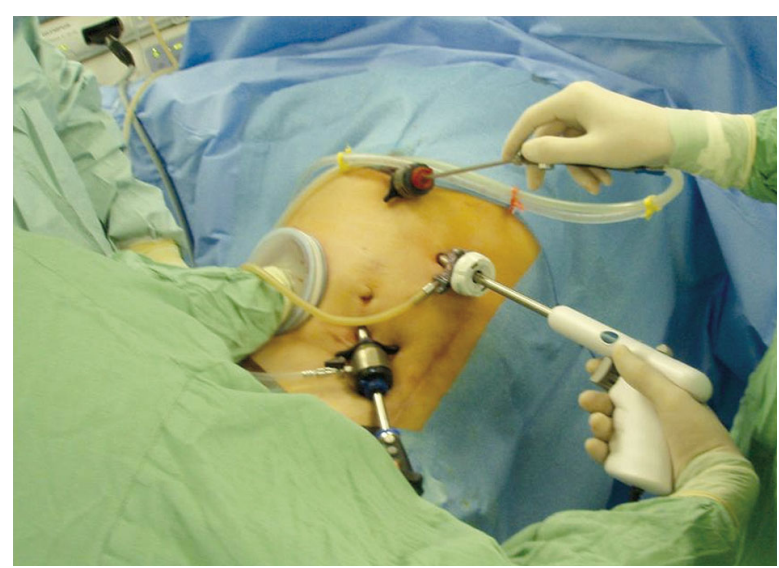

Figure 1. Intraoperative picture of positions of hand port and trocars 


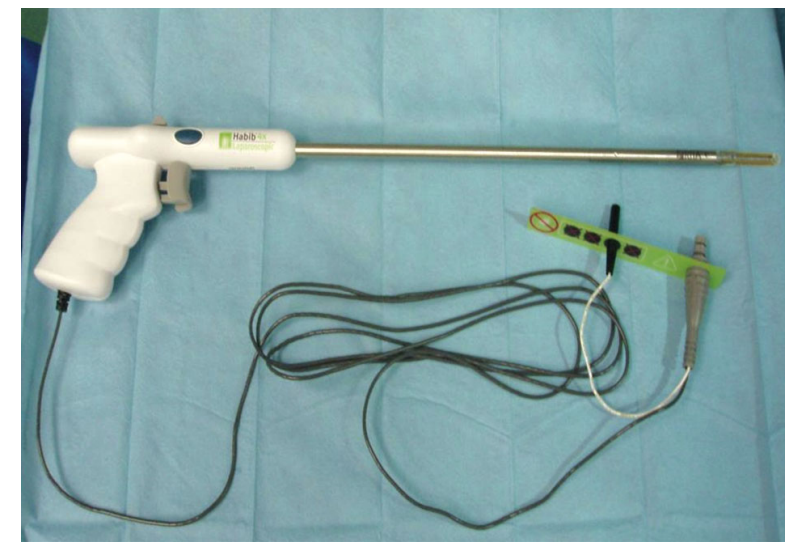

Figure 2. The laparoscopic Habib ${ }^{\text {TM }}$ 4X (bipolar device)

was made at the level of the umbilicus about $8 \mathrm{~cm}$ away from it. This quite low position of the hand port (Figure 1) helped us to achieve better control in liver mobilization and division of the right coronary ligament by giving more space for hand manoeuvring instead of putting the patient in the lateral decubitus position. Also this position of the hand port is convenient if a decision of conversion to open surgery is taken, so that this transverse incision is converted to a J-shaped incision.

Extensive liver mobilization should be avoided, and only the side to be resected should be mobilized for most cases. Once the tumour is localized, by manual palpation through the hand port and laparoscopic intraoperative liver ultrasound, the resection line is marked on the liver surface $1 \mathrm{~cm}$ from the tumour

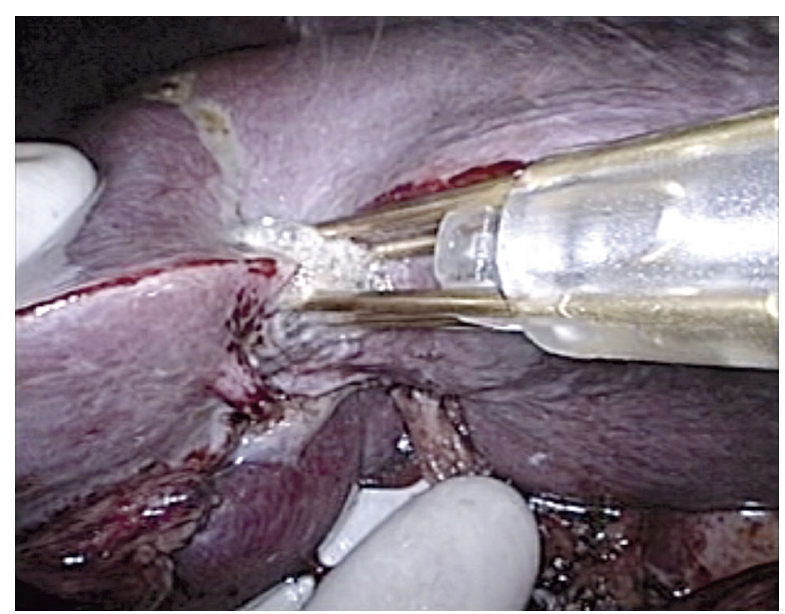

Figure 3. Hepatic parenchymal ablation using laparoscopic Habib 4X edge with diathermy. The marking of the transection line is very important, because after radiofrequency is used, the parenchyma is hardened and it becomes difficult to feel the tumour edge. Also, after radiofrequency intraoperative ultrasound fails to visualize the tumour edge due to the increased echogenicity resulting from radiofrequency (RF).

The laparoscopic Habib $4 \mathrm{X}$ is a bipolar device which consists of an array of four electrodes in a square arrangement (Figure 2). The electrodes are made of stainless steel, covered with a non-stick coating (Tomlinson Tube \& Instrument Ltd., Warwickshire, UK), with a polished titanium nitride nonstick coating (Tecvac Ltd., Cambridgeshire, UK; Integrated Surgical Sciences Corporation, Colorado, USA), to facilitate insertion and removal from the hepatic tissue. The four needles are arranged in a $2 \times 2$ array with the two pairs of needles connected together and each pair is connected to a single terminal of a bipolar radiofrequency generator (Generator 1500x, RITA Medical Systems Inc., California, USA) [11]. As we do not use any liver retractors, the hepatic parenchyma is supported by the surgeon's hand when the sealer is introduced perpendicularly into the liver, abutting the transaction line. For hepatic parenchymal coagulation the power of the generator is set at $100 \mathrm{~W}$. The power is lowered to $80 \mathrm{~W}$ or even $50 \mathrm{~W}$ for sealing larger vessels such as hepatic veins. Decreasing the power setting achieves a wider coagulation effect in a paradoxical manner.

During parenchymal ablation, the surgeon's hand is placed under the liver to protect the underlying structure (Figure 3). As the probe is withdrawn from the liver, the coagulated parenchyma is again supported by the surgeon's fingers from both sides in order to avoid fracturing leading to unnecessary blood loss and damage. The probe is introduced again adjacent to the last coagulated area, in a serial fashion, until the area to be transected is ablated. Preferably, a second line of ablation parallel to the first line is done to ensure complete tissue coagulation and perfect haemostasis prior to transection.

Next, laparoscopic scissors are introduced and used to divide the parenchyma near to the coagulated edge next to the tumour, leaving $10 \mathrm{~mm}$ of the coagulated edge. This allows a small, less than $10 \mathrm{~mm}$, margin of coagulated liver parenchyma to remain behind, ensuring sealed vessels and bile ducts. Less than $1 \mathrm{~cm}$ of tissue is left behind, just 


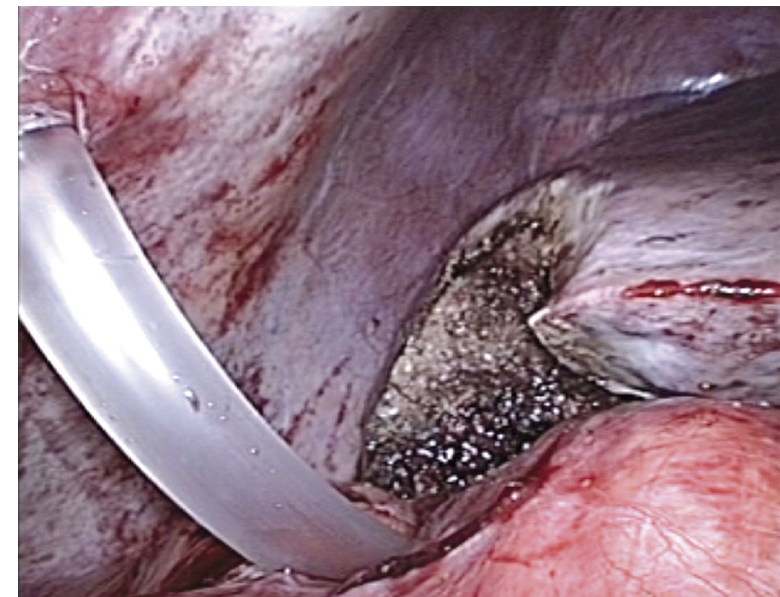

Figure 4. Liver bed after resection with a completely dry operative field

enough to ensure vascular and biliary sealing, yet not so much as to increase the rate of postoperative collections and/or abscess formation.

The resected part of the liver is retrieved through the hand port and sent for histopathological examination. Finally, mobilization of the omentum to the resection bed is done to minimize adhesions and facilitate mobilization for repeat resections if necessary. A $12 \mathrm{~F}$ silicon drain is placed through the $5 \mathrm{~mm}$ port site (Figure 4).

\section{Results}

Lesions were located in right inferior segments $V$ and VI and in left segments II and III. Tumours were solitary in all cases. As shown in Table II, the types of liver resection included metastasectomy $(n=7)$, segmentectomy $(n=5)$ and bisegmentectomy $(n=3)$.

All the procedures were achieved according to the previously described technique of hand-assisted laparoscopic radiofrequency-assisted liver resection. Haemostasis in all our patients was obtained only by radiofrequency energy; no additional devices (e.g. stitches, knots, clips or glue) were needed.

The average size of the resected tumour was $38 \mathrm{~mm}$ (range: $15-53 \mathrm{~mm}$ ). Total blood loss was $74 \mathrm{ml}$ (range: $25-115 \mathrm{ml}$ ). No patients received intraoperative or postoperative transfusion. The mean operative time was 92 min (range: 45-130 min), and the mean actual resection time was $33 \mathrm{~min}$ (range: 21-75 min). In 4 cases, laparoscopic cholecystectomy was also performed because of close proximity of the tumour to the gallbladder. Histopathological examination of

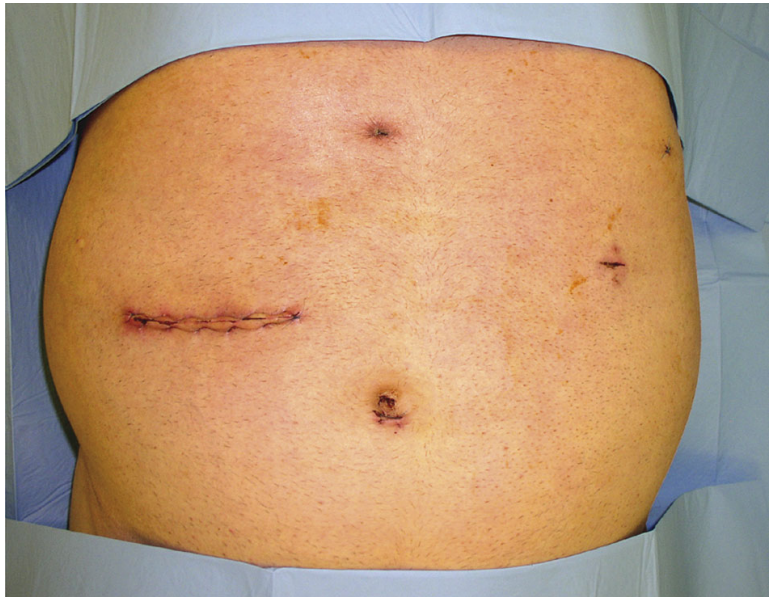

Figure 5. Cosmetic result at the end of the operation

the resected specimens confirmed RO resection in all cases.

Mean hospital stay after surgery was 5.1 days (range: 4-7 days). There were no major surgical complications and no postoperative mortality. We noted superficial wound infection in 1 patient. At mean follow-up of 16.5 months (range: 3.2-30.8 months), none of the patients with malignancy had developed recurrence at the resection margins.

\section{Discussion}

Hand-assisted laparoscopic liver resection has the additional advantage over conventional laparoscopy of preserving the surgeon's tactile palpation, together with the possibility of blunt dissection. It also offers an additional way of immediate control of bleeding if that happens and poses an oncological way of extracting malignant specimens.

The hand port system had disadvantages such as fatigue in the inserting hand which occurs if the operative time is prolonged [12]. In our experience, the potential benefit of reducing the operative time offered by using radiofrequency energy in parenchymal transaction without any time wasted in vascular inflow control or hilar dissection has eliminated this disadvantage.

An additional advantage of this procedure is the avoidance of major incisions (Figure 5) in patients with small tumours requiring limited resections, which subsequently reduces wound-related complications. 
Intraoperative blood loss remains a major concern for surgeons operating on the liver. It is associated with a higher rate of postoperative complications and shorter long-term survival. Over the years, different techniques have been developed to allow safer liver resection [13-16]. The technique for liver resection using RF energy was used to coagulate the liver resection margins. A $2 \mathrm{~cm}$ wide coagulative necrosis zone is created before division of the parenchyma with minimal blood loss.

The laparoscopic Habib $4 \mathrm{X}$ has the advantage over other laparoscopic radiofrequency devices used previously in liver resection by being a bipolar device. This increases the safety by eliminating the use of a ground pad electrode which may result in heat burns. In addition, the bipolar device is much faster than monopolar ones, which contributed to a decrease in the operating time of our case.

Our technique of hepatic resection was done without vascular clamping. Dissection of the hepatic pedicle was not performed and there was no need for extensive hepatic mobilization. The lesion in our case was in the periphery. Although we did not experience different tumour locations, we suggest that where the tumour is located in close proximity $(1 \mathrm{~cm}$ or less) to the important hilar structures, the separation of the tumour from the hilar structures with the classical Kellyclasia technique will be necessary without the use of the Habib $4 X$.

\section{Conclusions}

The combined benefits of the hand-assisted approach together with using radiofrequency for parenchymal transection might lead to a decrease in operative morbidity and mortality especially in highrisk patients such as the obese, by offering them a minimally invasive approach together with a safer technique of hepatic parenchymal transaction. This might encourage more surgeons to perform more frequently a laparoscopic approach for liver resection.

\section{Acknowledgments}

We declare that we have no personal, political or financial interest in the data or the technique described. All authors of this paper have read and approved the final version submitted. The contents of this manuscript have not been copyrighted or published previously.

\section{References}

1. Morino MI, Rosso E, Miglietta C, Garrone C. Laparoscopic vs open hepatic resection: a comparative study. Surg Endosc 2003; 17: 1914-8.

2. Vibert E, Perniceni T, Levard $H$, et al. Laparoscopic liver resection. Br J Surg 2006; 93: 67-72.

3. Buell JF, Thomas MJ, Doty TC, et al. An initial experience and evolution of laparoscopic hepatic resectional surgery. Surgery 2004; 136: 804-11.

4. Blumgart LH BJ. Liver resection for benign disease and for liver and biliary tumors. In: Surgery of the liver, biliary tract, and pancreas. Blumgart LH (ed). $4^{\text {th }}$ ed. Saunders, Philadelphia 2007; 1341-416.

5. Dostalik J, Martinek L, Mazur M, et al. Hand assisted laparoscopic living donor nephrectomy - initial experience. Surg Endosc 2004; 18: 128.

6. Dostalik J, Martinek L. Hand assisted laparoscopic colon surgery. 8th Biennial Congress European Council of Coloproctology 2001, Prague, Czech Republic.

7. Tang CN, Li MK. Laparoscopic-assisted liver resection. J Hepatobiliary Pancreat Surg 2002; 9: 105-10.

8. Fong Y, Jarnagin W, Conlon KC, et al. Hand assissted laparoscopic liver resection: lessons from an initial experience. Arch Surg 2000; 135: 854-9.

9. Weber JC, Navarra G, Jiao LR, et al. New technique for liver resection using heat coagulative necrosis. Ann Surg 2002; 236: 560-3.

10. Ayav A, Bachellier P, Habib NA, et al. Impact of radiofrequency assisted hepatectomy for reduction of transfusion requirements. Am J Surg 2007; 193: 143-8.

11. Healey A, Habib NA, Jiao LR. Liver resection assisted with the radiofrequency technique. In: Liver and biliary tract surgery. Karaliotas CC, Habib NA. 1st Ed. Springer, Athens, New York 2006; 367-72.

12. Huang M, Lee WJ, Wang W, et al. Hand assisted laparoscopic hepatectomy for solid tumors in the posterior portion of the right lobe initial experience. Ann Surg 2003; 238: 674-9.

13. Hansen PD, Isla AM, Habib NA. Liver resection using total vascular exclusion, scalpel division of the parenchyma and a simple compression technique for haemostasis and biliary control. J Gastrointest Surg 1999; 3: 537-42.

14. Nuzzo G, Guiliante F, Giovianni I, et al. Hepatic resections in normothermic ischemia. Surgery 1996; 120: 852-8.

15. Tranberg KG, Rigotti P, Brackett KA, et al. Liver resection. A comparison using Nd-YAG laser, an ultrasonic surgical aspirator, or blunt dissection. Am J Surg 1986; 151: 368-73.

16. Yamamoto Y, Ikai I, Kume M, et al. New technique for hepatic parenchymal resection using a Cavitron ultrasonic surgical aspirator and bipolar cautery equipped with a channel for water dripping. World J Surg 1999; 23: 1032-7. 